\title{
Síndrome de dificultad respiratoria vs síndrome de dificultad respiratoria por COVID-19: las diferencias que realmente importan
}

Respiratory distress syndrome vs COVID-19 respiratory distress syndrome: the differences that really matter Síndrome da dificuldade respiratória vs síndrome da dificuldade respiratória por COVID-19: as diferenças que realmente importam

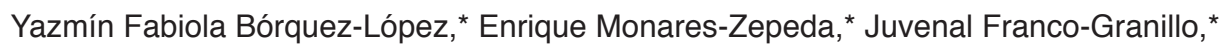
Janet Silvia Aguirre-Sánchez, ${ }^{*}$ Rodrigo Chaires-Gutiérrez*

\section{RESUMEN}

Introducción: El síndrome respiratorio agudo severo causado por el coronavirus 2 (SARS-CoV-2), ahora clasificado como enfermedad por coronavirus 2019 (COVID-19), ha presentado mayor morbilidad y mortalidad, en gran parte a causa de la neumonitis viral aguda que evoluciona a síndrome de dificultad respiratoria aguda (SDRA), pero pocos estudios han analizado las comparaciones entre ambas enfermedades.

Objetivos: Evaluar las características sociodemográficas, parámetros ventilatorios y mortalidad en pacientes con síndrome de dificultad respiratoria (ARDS) vs síndrome de dificultad respiratoria por COVID-19 (CARDS).

Material y métodos: Estudio de cohorte prospectivo en pacientes con ARDS vs CARDS bajo ventilación mecánica.

Resultados: 95 pacientes evaluados, 47 (49.4\%) presentaron ARDS y 48 (50.5\%) presentaron COVID-19. En la comparación de parámetros de oxigenación $\mathrm{PaO}_{2} / \mathrm{FiO}_{2}$ de $134 \mathrm{mmHg}$ (rango intercuartil $57-411 \mathrm{mmHg}$ ) en el grupo de ARDS y $118 \mathrm{mmHg}$ (rango intercuartil $50-351 \mathrm{mmHg}$ ) con una $p=0.746$ en CARDS sin diferencias significativas, en ambos grupos se respetaron medidas de protección pulmonar con volúmenes tidales bajos $6-8 \mathrm{~mL} / \mathrm{kg}$ peso ARDSnet en ARDS $5.8 \mathrm{~mL} / \mathrm{kg}$ (rango intercuartil 4.7-7.8) y CARDS $6.3 \mathrm{~mL} / \mathrm{kg}$ (Rango Intercuartil 4.5-7.5) $\mathrm{p}=0.51$, presión meseta $>30 \mathrm{mmHg}$, en CARDS de 24.6 $\pm 3.2 \mathrm{cmH}_{2} \mathrm{O}$ y ARDS $24.8 \pm 3.2 \mathrm{cmH}_{2} \mathrm{O}, \mathrm{p}=0.810$, presión de conducción $<15$ $\mathrm{mmHg}$, fue de $13.1 \pm 3.1 \mathrm{cmH}_{2} \mathrm{O}$ en ARDS y CARDS de $12.9 \pm 2.3 \mathrm{cmH}_{2} \mathrm{O} p=$ 0.73. Con mayor mortalidad $48.9 \%$ y días de ventilación mecánica en pacientes con ARDS.

Conclusión: No se encontraron diferencias significativas en ambos grupos respecto al índice de oxigenación, ambos respetaron metas de protección pulmonar, pero se encontró mayor mortalidad y días de ventilación mecánica en el grupo de ARDS.

Palabras clave: ARDS, CARDS, $\mathrm{PaO}_{2} / \mathrm{FiO}_{2}$, mortalidad.

\section{ABSTRACT}

Introduction: The severe acute respiratory syndrome caused by coronavirus 2 (SARS-CoV-2), now classified as coronavirus disease 2019 (COVID-19), has presented higher morbidity and mortality, largely to acute viral pneumonitis that evolves into syndrome respiratory distress (ARDS), but only few studies have analyzed comparisons between the two diseases.

Objectives: To evaluate the sociodemographic characteristics, ventilatory parameters and mortality in patients with ARDS vs CARDS.

Material and methods: Prospective cohort study in patients with ARDS vs CARDS under mechanical ventilation.

Results: 95 patients evaluated, 47 (49.4\%) presented ARDS and 48 (50.5\%) presented COVID-19. In the comparison of $\mathrm{PaO}_{2} / \mathrm{FiO}_{2}$ oxygenation parameters of $134 \mathrm{mmHg}$ (IQR $57-411 \mathrm{mmHg}$ ) in the ARDS group and $118 \mathrm{mmHg}$ (IQR $50-351 \mathrm{mmHg}$ ) with a $p=0.746$ in CARDS without significant differences, in both groups lung protection measures were respected with low tidal volumes 6-8 mL/kg ARDSnet weight in ARDS $5.8 \mathrm{~mL} / \mathrm{kg}$ (IQR 4.7-7.8) and CARDS 6.3 $\mathrm{mL} / \mathrm{kg}$ (IQR 4.5-7.5) $p=0.51$, Plateau pressure $>30 \mathrm{mmHg}$, in CARDS of 24.6

* Centro Médico ABC. Ciudad de México, México.

Recibido: 16/10/2020. Aceptado: 09/11/2020.

Citar como: Bórquez-López YF, Monares-Zepeda E, Franco-Granillo J, AguirreSánchez JS, Chaires-Gutiérrez R. Síndrome de dificultad respiratoria vs síndrome de dificultad respiratoria por COVID-19: las diferencias que realmente importan. Med Crit. 2021;35(4):176-181. https://dx.doi.org/10.35366/101155

www.medigraphic.com/medicinacritica
$\mathrm{CmH}_{2} \mathrm{O} \pm 3.2$ and $A R D S 24.8 \mathrm{cmH}_{2} \mathrm{O} \pm$ 3.2. $p=0.810$, conduction pressure $<15$ $\mathrm{mmHg}$, was $13.1 \mathrm{cmH}_{2} \mathrm{O} \pm 3.1 \mathrm{in}$ ARDS and CARDS of $12.9 \pm 2.3 \mathrm{cmH}_{2} \mathrm{O} p=$ 0.73 . With $48.9 \%$ higher mortality and days of mechanical ventilation in patients with ARDS.

Conclusion: No significant differences were found in both groups regarding the oxygenation index, both respected lung protection goals, but higher mortality and days of mechanical ventilation were found in the ARDS group.

Keywords: ARDS, CARDS, $\mathrm{PaO}_{2} / \mathrm{FiO}_{2}$, mortality.

\section{RESUMO}

Introdução: A síndrome respiratória aguda grave causada pelo coronavírus 2 (SARS-CoV-2), agora classificada como doença por coronavírus 2019 (COVID-19), apresentou maior morbimortalidade principalmente por pneumonite viral aguda que evolui ao síndrome do desconforto respiratório (ARDS), mas poucos estudos analisaram as comparações entre as duas doenças.

Objetivos: Avaliar as características sociodemográficas, parâmetros ventilatórios e mortalidade em pacientes com ARDS vs CARDS.

Material e métodos: Estudo de coorte prospectivo em pacientes com ARDS vs CARDS com ventilação mecânica.

Resultados: 95 pacientes avaliados, 47 (49.4\%) apresentaram ARDS e 48 (50.5\%) apresentaram COVID-19. Na comparação dos parâmetros de oxigenação $\mathrm{PaO}_{2} / \mathrm{FiO}_{2}$ de $134 \mathrm{mmHg}$ (RIC $57-411 \mathrm{mmHg}$ ) no grupo ARDS e $118 \mathrm{mmHg}$ (RIC 50-351 mmHg) com uma $p=0.746$ em CARDS sem diferenças significativas, em ambos os grupos as medidas de proteção pulmonar foram respeitadas com volumes correntes baixos $6-8 \mathrm{~mL} / \mathrm{kg}$ ARDSnet em ARDS 5.8 $\mathrm{mL} / \mathrm{kg}$ (RIC 4.7-7.8) e CARDS $6.3 \mathrm{~mL} / \mathrm{kg}$ ( RIC 4.5-7.5) $p=0.51$, pressão de platô $>30 \mathrm{mmHg}$, em CARDS de $24.6 \pm 3.2$ ARDS $24.8 \mathrm{cmH}_{2} \mathrm{O} \pm 3.2 p=$ 0.810 , pressão de condução $<15 \mathrm{mmHg}$, foi de $13.1 \mathrm{cmH}_{2} \mathrm{O} \pm 3.1 \mathrm{em}$ ARDS e CARDS de $12.9 \pm 2.3 \mathrm{CmH}_{2} \mathrm{O} p=0.73$. Com maior mortalidade $48.9 \%$ e dias de ventilação mecânica em pacientes com ARDS.

Conclusão: Não foram encontradas diferenças significativas nos dois grupos quanto ao índice de oxigenação, ambos respeitando as metas de proteção pulmonar, mas maior mortalidade e dias de ventilação mecânica foram encontrados no grupo SDRA.

Palavras-chave: ARDS, CARDS, $\mathrm{PaO}_{2} / \mathrm{FiO}_{2}$, mortalidade.

\section{INTRODUCCIÓN}

En 1967 se observó una revisión de 272 pacientes bajo ventilación mecánica que no respondían a tratamiento convencional, por lo que el Dr. Ashbaugh y colaboradores realizaron la primera descripción del síndrome de dificultad respiratoria (ARDS por sus siglas en inglés) que constaba de la descripción de 12 pacientes con cuadro clínico de taquipnea, disnea severa, acidosis respiratoria, hipoxemia refractaria, distensibilidades pulmonares bajas e infiltrados alveolares difusos por radiologías, en necropsias se encontró que todos los principales vasos pulmonares estaban permeables y libres de trombos o embolia, el árbol traqueobronquial estaba libre de obstrucción. Las características Ilamativas fueron dilatación, con- 
gestión de capilares y áreas de atelectasia alveolar, hemorragia y edema intersticial e intraalveolar, macrófagos alveolares y presencia de membranas hialinas. ${ }^{1}$

El síndrome de dificultad respiratoria aguda fue definido en 1994 por la Conferencia de Consenso Europeo-Estadounidense (AECC por sus siglas en inglés); desde entonces, han surgido problemas relacionados con la confiabilidad y validez de esta definición, porque se realizó un panel de expertos reunido en 2011 (una iniciativa de la Sociedad Europea de Medicina de Cuidados Intensivos respaldada por la Sociedad Americana del Tórax y la Sociedad de Medicina de Cuidados Críticos) donde se desarrolló la Definición de Berlín (Tabla 1), centrándose en la viabilidad, fiabilidad, validez, y evaluación objetiva de su desempeño, se evaluó empíricamente mediante un metaanálisis a nivel de paciente de 4,188 casos con ARDS de cuatro conjuntos de datos clínicos multicéntricos, y 269 pacientes con ARDS de tres conjuntos de datos de un solo centro que contienen información fisiológica. Los estadios de ARDS leve, moderado y grave se asociaron con un aumento de la mortalidad (27\%; IC del 95\%, 24-30\%; 32\%; IC del 95\%, 29-34\%; y 45\%; IC del 95\%, 42-48\%, respectivamente; $p<0.001$ ) y mayor duración media de la ventilación mecánica en los supervivientes (cinco días intercuartil [IQR] 2-11; siete días IQR, 4-14; y nueve días IQR, 5-17, respectivamente; $p<0.001)$. En comparación con la primer definición de AECC, la Definición de Berlín final tuvo una mejor validez predictiva para la mortalidad, con un área bajo la curva operativa del receptor de 0.577 (IC del 95\%, 0.561-0.593) frente a 0.536 (IC del 95\%, $0.520-0.553 ; p<0.001){ }^{2}$

\section{Fisiopatología del ARDS}

Se inicia la lesión por agresiones directas o indirectas a la delicada estructura alveolar del pulmón distal y la microvasculatura asociada. Por lo que se describen las siguientes fases:

Fase exudativa: los macrófagos alveolares residentes se activan, dando lugar a potentes mediadores proinflamatorios y quimiocinas que promueven la acumulación de neutrófilos y monocitos, esta activación contribuye aún más a la lesión al liberar mediadores tóxicos. La lesión resultante conduce a la pérdida de la función de barrera, así como a la inundación intersticial e intraalveolar. La expresión del factor tisular mediada por el factor de necrosis tumoral (TNF) promueve la agregación plaquetaria y la formación de microtrombos, así como la coagulación intraalveolar y la formación de membranas hialinas.

Fase proliferativa: tiene como objetivo restaurar la homeostasis tisular y se caracteriza por la expansión transitoria de los fibroblastos residentes y la formación de una matriz provisional, así como la proliferación de células progenitoras de las vías respiratorias y células epiteliales alveolares tipo II (ECAII), con diferenciación en células epiteliales alveolares tipo I (ECAI).

Fase fibrótica: está fuertemente asociada con la necesidad de ventilación mecánica, el daño extenso de la membrana basal, y la reepitelización inadecuada o retardada conducen al desarrollo de intersticial e intraalveolar fibrosis. ${ }^{3}$

La respuesta inflamatoria subsiguiente a la lesión subyacente provoca daño a las barreras epiteliales (exacerbado por el estiramiento mecánico) y acumulación de líquido de edema rico en proteínas en los alvéolos. Con el tiempo, se restablece la integridad epitelial y se reabsorbe el líquido alveolar. La fibrosis puede seguir y aumentar el riesgo de mortalidad. Fisiológicamente, el daño alveolar da como resultado un desajuste de ventilación-perfusión (desajuste de $V / Q$ ), como lo demuestran las observaciones de aumento de la derivación (alvéolos incapaces de intercambiar oxígeno) y espacio muerto (lesión microvascular que conduce a la falta de perfusión). . $^{2,3}$

Tomografía axial computarizada en ARDS: el diagnóstico de ARDS se realiza bajo tomografía posterior de cumplir con los criterios de definición de Berlín (Tabla 1).4

Tabla 1: Características demográficas y comorbilidades. $\mathrm{N}=95$.

\begin{tabular}{|c|c|c|c|c|}
\hline & $\mathrm{n}(\%)$ & $\begin{array}{c}\text { ARDS } \\
n=47 \\
n(\%)\end{array}$ & $\begin{array}{c}\text { CARDS } \\
n=48 \\
n(\%)\end{array}$ & $p$ \\
\hline Masculino ${ }^{2,3}$ & $51(53.7)$ & 18 (38.29) & $34(70.8)$ & 0.01 \\
\hline Edad (años) ${ }^{1,5}$ & $61(20-87)$ & $63(20-80)$ & $60(30-84)$ & 0.92 \\
\hline 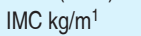 & $29.6(20-48.2)$ & 27.89 (22.67-48.2) & $28.35(22.8-34.4)$ & 0.595 \\
\hline Hipertensión ${ }^{2,3}$ & $32(33.7)$ & $7(14.8)$ & $26(54.1)$ & 0.47 \\
\hline Diabetes 2,3 & $28(29.7)$ & $15(31.9)$ & $13(27.0)$ & 0.60 \\
\hline $\mathrm{EPOC}^{2,4}$ & $11(11.7)$ & $4(8.3)$ & $7(14.58)$ & 0.52 \\
\hline $\operatorname{ICC}^{2,3}$ & $6(6.2)$ & 0 & $7(14.58)$ & 0.007 \\
\hline \multicolumn{5}{|l|}{ TAC } \\
\hline \multicolumn{5}{|c|}{ CORADS score ${ }^{2}$} \\
\hline 0 & $47(49.5)$ & & $47(49.5)$ & \\
\hline 1 & $3(3.2)$ & & $3(3.2)$ & \\
\hline 2 & $6(6.3)$ & $\mathrm{N} / \mathrm{A}$ & $6(6.3)$ & NS \\
\hline 3 & 17 (17.9) & & $17(17.9)$ & \\
\hline 4 & $12(12.6)$ & & $12(12.6)$ & \\
\hline 5 & $9(9.5)$ & & $9(9.5)$ & \\
\hline \multicolumn{5}{|c|}{ TAC ARDS score ${ }^{2}$} \\
\hline 1 & $5(10.6)$ & $5(10.6)$ & & \\
\hline 2 & $11(11.6)$ & $11(23.4)$ & & \\
\hline 3 & $17(17.6)$ & $17(36.1)$ & $\mathrm{N} / \mathrm{A}$ & NS \\
\hline 4 & $11(11.6)$ & $11(23.4)$ & & \\
\hline 5 & 3 (3.2) & $3(6.38)$ & & \\
\hline
\end{tabular}

ARDS = síndrome de dificultad respiratoria, $C A R D S=$ síndrome de dificultad respiratoria por COVID-19, IMC = índice de masa corporal, EPOC = enfermedad pulmonar obstructiva crónica, ICC = insuficiencia cardíaca crónica, TAC = tomografía axial computarizada,

${ }^{1}$ Rangos intercuartiles para variables de libre distribución variables cuantitativas.

${ }^{2}$ Desviación estándar para variables de distribución normal para variables cuantitativas.

${ }^{3}$ Frecuencias y proporciones variables cualitativas.

${ }^{4} \chi^{2}$ Pearson.

${ }^{5}$ Prueba exacta de Fisher.

${ }^{6} \mathrm{t}$ Student 
CI ARDS extrapulmonar la opacificación en vidrio esmerilado era más del doble de consolidación. Por el contrario, el ARDS pulmonar cuenta con un equilibrio entre opacificación y consolidación en vidrio deslustrado. La presencia de broncogramas aéreos y neumomediastino, lesiones de tipo enfisematorio (bullas) fueron comparables en ambos grupos. CI ARDS pulmonar se asocia con mayor frecuencia con pérdida difusa y en parches de aireación en tomografía computarizada (TC), mientras que ARDS extrapulmonar se acompaña más comúnmente de pérdida de aireación lobular.

\section{Fisiopatología del síndrome de dificultad respiratoria por COVID-19 (CARDS)}

EI SARS-CoV-2 es un coronavirus que utiliza la enzima convertidora de angiotensina II (ECAII) como fuente de entrada celular, al igual que los agentes coronavirus del SARS y el síndrome respiratorio de Oriente Medio (MERS). La ECAll se expresa en células alveolares pulmonares, epitelio bronquial y células endoteliales vasculares, lo que explica por qué el tracto respiratorio y el pulmón sirven como punto primario de entrada viral. ${ }^{5,6}$

Se han reportado en análisis patológicos pulmones congestionados y edematosos con afectación parcheada y área de consolidación difusa. ${ }^{5-7}$

En $92 \%$ (35/38) de los pacientes se presentaron en las vías respiratorias superiores (tráquea y bronquios grandes) manchas blancas focales, que histológicamente mostraban ulceración de la mucosa con infiltración de células inflamatorias mixtas incluyendo neutrófilos y fibrina. La evaluación microscópica de los pulmones en diferentes áreas anatómicas (vías respiratorias, alvéolos y lecho vascular) para los patrones de lesión mostraban presencia frecuente de inflamación de las vías respiratorias y zonas alveolares con membranas hialinas e hiperplasia de neumocitos tipo 2 (células AT2). Se encontraron con menos frecuencia proliferación fibroblástica intersticial con estroma fibromixoide, neumonía organizada, metaplasia escamosa e inflamación aguda de la pared alveolar. La hiperplasia de células AT2 y la proliferación fibroblástica intersticial tenían más probabilidades de identificarse con una enfermedad de mayor duración. Nueve pacientes sin membranas hialinas, cinco tenían inflamación aguda de las paredes alveolares, tres tenían émbolos en silla de montar o de la arteria pulmonar principal y uno tenía enfermedad cardiaca y escasa afectación pulmonar. Los neumocitos con frecuencia aparecían atípicos, agrandados y, a veces, multinucleados con características sincitiales. La lesión capilar y necrosis se observó en un subconjunto de casos, lo que sugiere una endotelitis. ${ }^{7,8}$

Diferencias de CARDS: Gattinioni L y colaboradores proponen dos tipos de pacientes con neumonía por COVID-199-11
Tipo 1 (tipo L): pacientes con hipoxemia con distensibilidad pulmonar $>50 \mathrm{~mL} / \mathrm{cmH}_{2} \mathrm{O}$, con capacidad de reclutamiento pulmonar mínima, probable secundaria a la pérdida de la vasoconstricción pulmonar hipóxica y regulación alterada del pulmón, por lo que el principal mecanismo de hipoxemia se ve en relación con la ventilación/ perfusión, por lo que la presión positiva al final de la espiración (PEEP) y la posición prono no mejoran la oxigenación mediante reclutamiento de áreas colapsadas, sino redistribuyendo la perfusión pulmonar. Los pacientes que se clasifican en esta categoría por las características pulmonares se pueden mantener con volumen tidal de 7-8 m/ $\mathrm{kg}$ ARDS, y frecuencias respiratorias de aproximadamente 15 a 20 respiraciones por minuto, se establece que los niveles de PEEP deben limitarse a 8 a $10 \mathrm{cmH}_{2} \mathrm{O}$, ya que niveles más altos pueden producir sobredistensión pulmonar y/o interacción cardiopulmonar. ${ }^{9-11}$

Tipo 2 (tipo H): aproximadamente 20 a $30 \%$ de los pacientes presentan hipoxemia grave, con distensibilidad pulmonar $<40 \mathrm{~mL} / \mathrm{cmH}_{2} \mathrm{O}$. En estos pacientes un aumento gradual cauteloso de la PEEP hasta 14-15 $\mathrm{CmH}_{2} \mathrm{O}$ puede ser beneficioso. ${ }^{9-11}$

Tomografía axial computarizada en CARDS: el papel de la TC de tórax en COVID-19 está en constante evolución con evidencia científica modesta pero diferencias sustanciales en la opinión sobre cuándo y cómo se debe utilizar la técnica para la evaluación clínica o las decisiones de tratamiento; COVID-19 presenta hallazgos de TC que se superponen parcialmente con otras enfermedades, en especial infecciones virales, pero también muestra rasgos característicos que se ven con menos frecuencia en otros entornos. ${ }^{12}$

CO-RADS (a categorical CT assessment scheme for patients suspected of having COVID-19) proporciona un nivel de sospecha de afectación pulmonar de COVID-19 según las características observadas en una TC de tórax no realizada. El nivel de sospecha aumenta de muy bajo (CO-RADS 1) a muy alto (CO-RADS 5). Dos categorías adicionales codifican, respectivamente un examen técnicamente insuficiente (CO-RADS 0) y una infección por SARS-CoV-2 probada por RT-PCR en el momento del examen (CO-RADS 6) (Tabla 2). ${ }^{13}$

\section{MATERIAL Y MÉTODOS}

Se trata de un estudio retrospectivo, longitudinal, de cohorte el cual se llevó a cabo en el Departamento de Terapia Intensiva «Dr. Mario Shapiro» en la Ciudad de México. El comité de ética institucional revisó y aprobó el presente estudio con número $\mathrm{ABC}-20-85$.

Se incluyeron 95 pacientes mayores de 18 años con diagnósticos de síndrome de dificultad respiratoria de marzo del 2019 a febrero 2020 y síndrome de dificultad respiratoria secundaria al virus SARS-CoV-2, que ingresaron a terapia intensiva bajo ventilación mecá- 
Tabla 2: Criterios tomográficos para COVID-19 (CO-RADS score).

\begin{tabular}{cll}
\hline CO-RADS & $\begin{array}{c}\text { Nivel de sospecha de afectación } \\
\text { pulmonar de COVID-19 }\end{array}$ & \multicolumn{1}{c}{ Resumen } \\
\hline 0 & No interpretable & $\begin{array}{l}\text { Escaneo técnicamente insuficiente para } \\
\text { asignar una puntuación }\end{array}$ \\
1 & Muy bajo & Normal o no infección \\
2 & Bajo & Típico de otra infección, pero no COVID-19 \\
3 & Equivocado/insegura & Lesiones compatibles con COVID-19 pero \\
& también con otras enfermedades \\
4 & Alto & Sospechoso de COVID-19 \\
5 & Muy alto & Típico de COVID-19 \\
6 & Prueba & PCR SARS-CoV-2 \\
\hline
\end{tabular}

PCR = reacción en cadena de la polimerasa

nica en el periodo de marzo del 2020 al 30 de agosto del 2020.

Excluimos pacientes con tiempo estimado de vida menor a tres meses, pacientes con enfermedad neoplásica en etapa terminal.

Todos los casos fueron reclutados desde el ingreso a UCl y seguidos hasta el egreso de Terapia Intensiva.

Recolección de los datos: al ingreso a la Unidad de Terapia Intensiva, todos los pacientes fueron evaluados para la obtención de las características demográficas como edad, género, índice de masa corporal (IMC) y enfermedades previas. Escalas pronósticas (SOFA, APACHE II, SAPS II), parámetros ventilatorios y de oxigenación.

Definición de desenlaces: el desenlace primario en este trabajo fue el grado de oxigenación, la cual definimos como niveles de $\mathrm{PaO}_{2} / \mathrm{FiO}_{2}$ y mecánica respiratoria expresadas con medidas de protección alveolar (presión meseta, volumen tidal, presión de conducción [Driving pressure]), niveles de PEEP. Los desenlaces secundarios incluyeron días de ventilación mecánica, días de estancia en UCl y mortalidad hospitalaria.

Análisis estadístico: las variables cualitativas se reportan como frecuencias y proporciones, las cuantitativas como mediana con rango intercuartílico o media con desviación estándar, dependiendo la normalidad de su distribución. Para la evaluación comparativa, si las variables eran cuantitativas se utilizó t de Student o U de Mann-Whitney, lo cual dependió de la evaluación previa de normalidad mediante Shapiro-Wilk o Kolmogorov-Smirnov. Para las variables cualitativas, se utilizó $\chi^{2}$ o prueba exacta de Fisher, si en la evaluación previa del valor esperado se encontró o no un valor $\leq 5$. Un valor de $\mathrm{p}$ menor o igual a 0.05 se consideró estadísticamente significativa.

\section{RESULTADOS}

De los 95 pacientes adultos incluidos en este estudio, $47(49.4 \%)$ presentaron ARDS y $48(50.5 \%)$ presentaron COVID-19, 53.7\% eran hombres, 56\% con mayor proporción de comorbilidades de hipertensión arterial, insuficiencia cardiaca congestiva $14.5 \%$ con $p=0.007$ en pacientes con CARDS. En la Tabla 1 se presentan las características de los casos estudiados por grupos.

Los pacientes con ARDS tuvieron un SOFA score mayor a 13 (RIC 0-16) a comparación de CARDS con 5 (RIC 1-12) con $p=0.00$, en SAPS II y APACHE II no presentaron diferencias estadísticamente significativas (Tabla 3), el requerimiento de vasopresores e inotrópicos fue mayor en el grupo CARDS con 85.41 y $14.58 \%$. La maniobra prono en mayor proporción $72.9 \%$ con $p=$ 0.000 en el grupo CARDS.

En la comparación de parámetros de oxigenación no presentaron diferencias significativas entre ambos grupos con niveles de oxigenación muy parecidos, en ambos grupos se respetaron medidas de protección pulmonar con volúmenes tidales bajos $6-8 \mathrm{~mL} / \mathrm{kg}$ peso ARDS network (ARDSnet) en ARDS $5.8 \mathrm{~mL} / \mathrm{kg}$ (RIC 4.7-7.8) y CARDS $6.3 \mathrm{~mL} / \mathrm{kg}$ (RIC 4.5-7.5) $\mathrm{p}=0.51$.

Presión meseta $>30 \mathrm{mmHg}$, en CARDS de $24.6 \pm 3.2$ $\mathrm{cmH}_{2} \mathrm{O}$ y ARDS $24.8 \pm 3.2 \mathrm{cmH}_{2} \mathrm{O}, \mathrm{p}=0.810$, presión de conducción $<15 \mathrm{mmHg}$ fue de $13.1 \pm 3.1 \mathrm{cmH}_{2} \mathrm{O}$ en ARDS y CARDS de $12.9 \pm 2.3 p=0.73$ (Tabla 4).

Los días de estancia en UCl fueron similares entre ambos grupos. Con mayor mortalidad y días de ventilación mecánica en pacientes con ARDS (Tabla 5).

\section{DISCUSIÓN}

Se trata de un estudio de cohorte prospectivo, en el cual evaluamos 95 pacientes con diagnóstico de neumonía grave por COVID-19 u otra etiología bajo ventilación mecánica. El objetivo principal fue evaluar la diferencia de oxigenación entre ambos grupos.

Dreher $\mathrm{M}$ y colaboradores en Alemania estudiaron 50 pacientes, de los cuales 26 no presentaron CARDS y 24 con CARDS con diferentes grados de severidad,

Tabla 3: Escalas de gravedad de Unidad de Cuidados Intensivos y tratamientos. $\mathrm{N}=95$.

\begin{tabular}{lrccl}
\hline & & ARDS & CARDS & $p$ \\
\hline SAPS II, media (DE) & $24(5-49)$ & $25(7-49)$ & $23(5-49)$ & 0.128 \\
APACHE II, mediana (RI) & $10(4-25)$ & $12(4-25)$ & $10(4-25)$ & 0.287 \\
SOFA, mediana (RI) & $8(0-16)$ & $13(0-16)$ & $5(1-12)$ & 0.000 \\
Vasopresores 4 , n (\%) & $76(80.0)$ & $35(74.4)$ & $41(85.41)$ & 0.18 \\
Inotrópicos $^{5}, \mathrm{n}(\%)$ & $11(11.6)$ & $4(8.5)^{5}$ & $7(14.58)$ & 0.523 \\
Prono $^{4}, \mathrm{n}(\%)$ & $45(46.9)$ & $10(21.27)$ & $35(72.9)$ & $0.000^{4}$ \\
\hline
\end{tabular}

ARDS = síndrome de dificultad respiratoria, CARDS = síndrome de dificultad respiratoria por COVID-19, SAPS = Simplified Acute Physiology Score, APACHE = Acute Physiology and Chronic Health Evaluation, SOFA = Sequential Organ Failure Assessment.

${ }^{1}$ Rangos intercuartiles para variables de libre distribución variables cuantitativas.

2 Desviación estándar para variables de distribución normal para variables cuantitativas.

${ }^{3}$ Frecuencias y proporciones variables cualitativas.

${ }^{4} \chi^{2}$ Pearson.

${ }^{5}$ Prueba exacta de Fisher.

${ }^{6}$ t Student

${ }^{7}$ U Mann-Whitney. 
Tabla 4: Desenlaces primarios de oxigenación y parámetros ventilatorios. $\mathrm{N}=95$.

\begin{tabular}{|c|c|c|c|c|}
\hline & & ARDS & CARDS & $\mathrm{p}$ \\
\hline Peso ARDSNET (mL/kg) & $6.1(4.5-7.8)$ & $5.8(4.7-78.35)$ & $6.3(4.5-7.5)$ & 0.051 \\
\hline $\mathrm{PaO}_{2} / \mathrm{FiO}_{2} \mathrm{mmHg}^{1,5}$ & $122(50-411)$ & $134(57-411)$ & $118(50-351)$ & 0.746 \\
\hline Volumen tidal 2,5 & $429 \pm 81.46$ & $434 \pm 98.7$ & $424 \pm 58.91$ & 0.53 \\
\hline Volumen minuto & $10(6.4-16)$ & $11(6.4-16)$ & $10(6.6-15)$ & 0.356 \\
\hline Presión pico $\mathrm{CmH}_{2} \mathrm{O}^{1}$ & $29(18-45)$ & $28(18-45)$ & $29(23-34)$ & 0.236 \\
\hline Presión media $\mathrm{cmH}_{2} \mathrm{O}^{2,3}$ & $19 \pm 4.53$ & $19.7 \pm 3.4$ & $18.51 \pm 5.4$ & 0.201 \\
\hline Presión meseta $\mathrm{CmH}_{2} \mathrm{O}^{2,4}$ & $24 \pm 4.14$ & $24.8 \pm 3.2$ & $24.6 \pm 3.2$ & 0.810 \\
\hline Presión de conducción 2,3 & $13 \pm 2.7$ & $13.1 \pm 3.1$ & $12.9 \pm 2.3$ & 0.73 \\
\hline PEEP $\mathrm{cmH}_{2} \mathrm{O}^{2,3}$ & $12 \pm 3.7$ & $10 \pm 4.35$ & $12 \pm 2.97$ & 0.78 \\
\hline Distensibilidad ${ }^{1,5}$ & $37(19-60)$ & $39(22-55)$ & $34.5(19-60)$ & 0.134 \\
\hline
\end{tabular}

ARDS = síndrome de dificultad respiratoria, CARDS = síndrome de dificultad respiratoria por COVID-19, $\mathrm{PaO}_{2}=$ presión parcial de oxígeno arterial, $\mathrm{FiO}_{2}=$ fracción de oxígeno inspirado, PEEP = presión respiratoria final positiva.

${ }^{1}$ Rangos intercuartiles para variables de libre distribución variables cuantitativas.

${ }^{2}$ Desviación estándar para variables de distribución normal para variables cuantitativas

${ }^{3}$ Frecuencias y proporciones variables cualitativas

${ }^{4} \chi^{2}$ Pearson

${ }^{5}$ Prueba exacta de Fisher

todos los casos tenían comorbilidades (hipertensión arterial, obesidad). En el grupo de CARDS, $71 \%$ requirió ventilación compleja en decúbito prono y $46 \%$ requirió hemodiálisis por insuficiencia renal aguda, ocho de los casos requirieron oxigenación por membrana extracorpórea (ECMO), la terapia con antibióticos se administró a 83\%; comparado con la presente investigación, las comorbilidades en los pacientes con CARDS fueron hipertensión $26 \%$, diabetes mellitus 13\%, EPOC $14.5 \%$ e insuficiencia cardiaca en $14.5 \%$, este último con diferencia significativa $p=0.007$, y que comparado con los pacientes con ARDS sólo se encontró mayor proporción en diabéticos (Tabla 1).4,5,14

Encontramos una mayor proporción de casos con requerimiento de vasopresores en el grupo de CARDS con el $85.41 \%$ y uso de inotrópicos $14.58 \%$ sin significancia estadística $p=0.18$ y $p=0.53$, pero en contraste observamos que los pacientes con CARDS ingresan con puntación de escala SOFA de severidad de 5 (RIC 1-12) a comparación de ARDS, con 13 (RIC 0-16), con diferencia significativa de $p=0.000$; además se observó que casos con CARDS tenían mayor proporción de realizar maniobra prono $72.9 \%$ con $p=0.000 . .^{10-12,15}$

Gattinioni $L$ y su grupo consideran que, a pesar de que se cumpla la definición de ARDS de Berlín en neumonía severa por COVID-19, se encuentra una disociación peculiar entre la gravedad de la hipoxemia y el mantenimiento de una mecánica respiratoria relativamente buena. Por lo que proponen dos tipos de pacientes con neumonía por COVID-19, en este estudio no se encontró diferencia entre nivel de oxigenación con una mediana de $\mathrm{PaO}_{2} / \mathrm{FiO}_{2}$ de $134 \mathrm{mmHg}$ (RIC $57-411 \mathrm{mmHg}$ ) en el grupo de ARDS y $118 \mathrm{mmHg}$ (RIC 50-351 mmHg) con una $p=0.746$ en CARDS; en cuanto a la mecánica respiratoria y parámetros de protección pulmonar diferenciado en pacientes tipo 1 ( $T L$ ), hipoxemia con distensibilidad pulmonar $>50 \mathrm{~mL} /$ $\mathrm{cmH}_{2} \mathrm{O}$, con capacidad de reclutamiento pulmonar mínima, vasoconstricción pulmonar y PEEP limitados a 8-10 $\mathrm{cmH}_{2} \mathrm{O}$ y tipo $2(\mathrm{TH})$, aproximadamente 20 a $30 \%$ presentan hipoxemia grave, con distensibilidad pulmonar $<40 \mathrm{~mL} / \mathrm{cmH}_{2} \mathrm{O}$. En estos pacientes un aumento gradual cauteloso de la PEEP hasta $14-15 \mathrm{cmH}_{2} \mathrm{O}$ secundarios por CARDS y casos con ARDS con criterios de Berlín (Tabla 6), en este estudio no se encontraron diferencias estadísticamente significativas entre ambas etiologías con peso ARDSnet en ARDS $5.8 \mathrm{~mL} /$ $\mathrm{kg}$ (RIC 4.7-7.8) y CARDS $6.3 \mathrm{~mL} / \mathrm{kg}$ (RIC 4.5-7.5) $\mathrm{p}$ $=0.51$, respetando la primera regla de volúmenes corrientes bajos.

Presión meseta CARDS $24.6 \pm 3.2 \mathrm{cmH}_{2} \mathrm{O}$ y ARDS $24.8 \pm 3.2 \mathrm{cmH}_{2} \mathrm{O}$ con presiones mesetas $<30 \mathrm{cmH}_{2} \mathrm{O}$ en ambos grupos con $p=0.810$, presión de conducción $13.1 \pm 3.1 \mathrm{cmH}_{2} \mathrm{O}$ ARDS y en CARDS $12.9 \pm 2.3, \mathrm{p}=$ 0.73 , y PEEP en ARDS $10 \pm 4.35 \mathrm{cmH}_{2} \mathrm{O}$ y CARDS 12 $\pm 2.97 \mathrm{cmH}_{2} \mathrm{O}, \mathrm{p}=0.78$, el resto de parámetros ventilatorios se muestran en la Tabla 4, donde en ninguno se encontró diferencia significativa entre las dos patologías con niveles de PEEP muy similares, y en todos se respetaron las medidas de protección alveolar.

Se encontró mayor mortalidad de los pacientes en ARDS $48.9 \%$ en este estudio comparado con CARDS $22.9 \%$ con diferencia significativa $\mathrm{p}=0.008$, así como días de ventilación mecánica en ARDS 12 (RIC 2-39) y CARDS ocho (4-22) con $\mathrm{p}=0.001$ (Tabla 5). ${ }^{16}$

\section{CONCLUSIÓN}

En el análisis de los grupos se encontraron pocas diferencias estadísticamente significativas entre ambos, de las cuales destacan en el grupo de CARDS comorbilidades más relacionadas a alteraciones cardiovasculares, SOFA score, no existe diferencia significativa entre grados de oxigenación y en ambos grupos se respetaron medidas de protección pulmonar, se presentó mayor mortalidad y días de ventilación mecánica en los pacientes con ARDS, probablemente por tratarse de una pandemia y una nueva enfermedad con pocos cono-

Tabla 5: Desenlaces secundarios. $\mathrm{N}=95$.

\begin{tabular}{lllrr}
\hline & & ARDS & CARDS & $p$ \\
\hline Mortalidad, $n(\%)^{2,3}$ & $34(35.8)$ & $23(48.9)$ & $11(22.9)$ & 0.008 \\
Días de estancia (UCl), mediana (RI) $)^{1,4}$ & $21(15-30)$ & $22(3-95)$ & $21(1-41)$ & 0.952 \\
Días de ventilación, mediana (RI) $)^{1,4}$ & $10(1-30)$ & $12(2-39)$ & $8(4-22)$ & 0.001 \\
\hline
\end{tabular}

ARDS = síndrome de dificultad respiratoria, $C A R D S=$ síndrome de dificultad respiratoria por COVID-19, UCl = Unidad de Cuidados Intensivos.

${ }^{1}$ Rangos intercuartiles para variables de libre distribución variables cuantitativas.

${ }^{2}$ Desviación estándar para variables de distribución normal para variables cuantitativas

${ }^{3}$ Frecuencias y proporciones variables cualitativas

${ }^{4} \chi^{2}$ Pearson 
Tabla 6: Criterios de Berlín para diagnóstico de ARDS.

\begin{tabular}{|c|c|}
\hline $\begin{array}{l}\text { Radiografía de tórax o tomografía } \\
\text { computarizada }\end{array}$ & $\begin{array}{l}\text { Opacidades bilaterales que no se explican } \\
\text { completamente por derrames pleurales, } \\
\text { colapso pulmonar o nódulos }\end{array}$ \\
\hline Etiología del edema & $\begin{array}{l}\text { No se explica completamente por insufi- } \\
\text { ciencia cardiaca o sobrecarga de volumen }\end{array}$ \\
\hline Sincronización & $\begin{array}{l}\leq \text { una semana desde: } \\
\text { - Síntomas respiratorios nuevos o } \\
\text { que empeoran y/o } \\
\text { - Insulto clínico conocido }\end{array}$ \\
\hline \multicolumn{2}{|l|}{ Oxigenación* (con PEEP $\geq 5 \mathrm{cmH}_{2} \mathrm{O}$ ) } \\
\hline \multicolumn{2}{|l|}{$\operatorname{SDRA}\left(\mathrm{PaO}_{2} / \mathrm{FiO}_{2}\right)$} \\
\hline Leve & $200-300 \mathrm{mmHg}$ \\
\hline Moderado & $100-200 \mathrm{mmHg}$ \\
\hline Severo & $\leq 100 \mathrm{mmHg}$ \\
\hline \multicolumn{2}{|l|}{ Mortalidad a los 90 días (\%) } \\
\hline 27 & (IC 95\%; 24-30\%) \\
\hline 32 & (IC 95\%; 29-34\%) \\
\hline 45 & (IC 95\%; 42-48\%) \\
\hline
\end{tabular}

* La presión positiva se puede administrar en forma no invasiva en el grupo de SDRA leve con presión positiva continua en las vías respiratorias (CPAP). $\mathrm{FiO}_{2}=$ fracción de oxígeno inspirado, $\mathrm{PaO}_{2}=$ presión parcial de oxígeno arterial, $\mathrm{PEEP}=$ presión respiratoria final positiva.

cimientos respecto a un tratamiento específico; como médicos, partimos del tratamiento y manejo de mecánica ventilatoria que tenemos del ARDS con más estudios que avalan mejor supervivencia y menor daño a los pacientes. Aun así, se requieren de estudios multicéntricos con un mayor número de casos para confirmar estos hallazgos.

\section{REFERENCIAS}

1. Ashbaugh DG, Bigelow DB, Petty TL, Levine BE. Acute respiratory distress in adults. Lancet. 1967;2(7511):319-323. doi: 10.1016/s0140-6736(67)90168-7.

2. Ferguson ND, Fan E, Camporota L, Antonelli M, Anzueto A, Beale R, et al. The Berlin definition of ARDS: an expanded rationale, justification, and supplementary material. Intensive Care Med. 2012;38:1573-1582.

3. Thompson BT, Chambers RC, Liu KD. Acute respiratory distress syndrome. N Engl J Med. 2017;377(6):562-572. doi: 10.1056/ NEJMra1608077.

4. Kamo T, Tasaka S, Suzuki T, Asakura T, Suzuki S, Yagi K, et al. Prognostic values of the Berlin definition criteria, blood lactate level, and fibroproliferative changes on high-resolution computed tomography in ARDS patients. BMC Pulm Med. 2019;19:37. Available in: https://doi.org/10.1186/s12890-0190803-0
5. Guan WJ, Ni ZY, Hu Y, Liang WH, Ou CQ, He JX, et al. Clinical characteristics of coronavirus disease 2019 in China. N Engl J Med. 2020;382:1708-1720. https://doi.org/10.1056/NEJMoa2002032

6. Joly BS, Siguret V, Veyradier A. Understanding pathophysiology of hemostasis disorders in critically ill patients with COVID-19. Intensive Care Mediciner. 2020;46:1063-1606. Available in: https://doi.org/10.1007/s00134-020-06088-1

7. Ackermann M, Verleden SE, Kuehnel M, Haverich A, Welte T, Laenger F, et al. Pulmonary vascular endothelialitis, thrombosis, and angiogenesis in Covid-19. N Engl J Med. 2020;383:120-128. Available in: https://doi.org/10.1056/NEJMoa2015432

8. Borczuk AC, Salvatore SP, Seshan SV, Patel SS, Bussel JB, Mostyka $\mathrm{M}$, et al. COVID-19 pulmonary pathology: a multi-institutional autopsy cohort from Italy and New York City. Mod Pathol. 2020; Available in: https://doi.org/10.1038/s41379-020-00661-1

9. Gattinoni L, Chiumello D, Caironi P, Busana M, Romitti F, Brazzi L, et al. COVID-19 pneumonia: different respiratory treatments for different phenotypes? Intensive Care Med. 2020;46:10991102. Available in: https://doi.org/10.1007/s00134-020-06033-2

10. Gattinoni L, Chiumello D, Rossi S. COVID-19 pneumonia: ARDS or not? Crit Care. 2020;24:154. Available in: https://doi. org/10.1186/s13054-020-02880-z

11. Li X, Ma X. Acute respiratory failure in COVID-19: is it "typical" ARDS? Crit Care. 2020;24:198. Available in: https://doi. org/10.1186/s13054-020-02911-9

12. Kunhua L, Jiong W, Faqi W, Dajing G, Linli C, Zheng F, et al. The clinical and chest CT features associated with severe and critical COVID-19 pneumonia. Invest Radiol. 2020;55(6):327-331. doi: 10.1097/RLI.0000000000000672.

13. Prokop M, Van Everdingen W, Van Rees VT, Quarles VH, Stoger L, Beenen L, et al. CO-RADS-A categorical CT assessment scheme for patients with suspected COVID-19: definition and evaluation. Radiology. 2020; 296:E97-E104. doi: 10.1148/radiol.2020201473.

14. Quah P, Li A, Phua J. Mortality rates of patients with COVID-19 in the intensive care unit: a systematic review of the emerging literature. Crit Care. 2020;24:285. Available in: https://doi. org/10.1186/s13054-020-03006-1

15. Dreher M, Kersten A, Bickenbach J, Balfanz, P, Hartmann B, Cornelissen $C$, et al. The characteristics of 50 hospitalized COVID-19 patients with and without ARDS. Dtsch Arztebl Int. 2020;117(16):271-278.

16. Valente-Acosta B, Hoyo-Ulloa I, Espinosa-Aguilar L, MendozaAguilar R, Garcia-Guerrero J, Ontañon-Zurita D, Gomez-Gomez $\mathrm{B}$, et al. COVID-19 severe pneumonia in Mexico City-First experience in a Mexican hospital. MedRxiv. 2020; Available in: https://doi.org/10.1101/2020.04.26.20080796

Conflicto de intereses: No tenemos ningún conflicto de intereses.

Correspondencia:

Enrique Monares Zepeda

E-mail: faby_borquez@hotmail.com 